\title{
Large Area Deposition of Field Emission Cathodes for Flat Panel Displays
}

\author{
Alan Jankowski \\ Jeffrey Hayes \\ Jeffrey Morse \\ James Ferreira
}

This paper was prepared for submittal to the

International Conference on Metallurgical Coatings and Thin Films

San Diego, CA

April 11-16, 1999

February 11, 1999

This is a preprint of a paper intended for publication in a journal or proceedings. Since changes may be made before publication, this preprint is made available with the understanding that it will not be cited or reproduced without the permission of the author. 


\section{DISCLAIMER}

This document was prepared as an account of work sponsored by an agency of the United States Government. Neither the United States Government nor the University of California nor any of their employees, makes any warranty, express or implied, or assumes any legal liability or responsibility for the accuracy, completeness, or usefulness of any information, apparatus, product, or process

disclosed, or represents that its use would not infringe privately owned rights. Reference herein to any specific commercial product, process, or service by trade name, trademark, manufacturer, or otherwise, does not necessarily constitute or imply its endorsement, recommendation, or favoring by the United States Government or the University of California. The views and opinions of authors expressed herein do not necessarily state or reflect those of the United States Government or the University of California, and shall not be used for advertising or product endorsement purposes. 


\title{
Large Area Deposition of Field Emission Cathodes for Flat Panel Displays
}

\author{
Alan Jankowski, Jeffrey Hayes, Jeffrey Morse, and James Ferreira \\ University of California - Lawrence Livermore National Laboratory \\ P.O. Box 808, Livermore CA 94550
}

\begin{abstract}
The convention for field emission cathode (FEC) synthesis involves coating with a veryhigh tolerance in thickness uniformity using a planetary substrate fixture and a large source-tosubstrate seperation. New criteria for a deposition process must facilitate a reduction in the operating voltage by increasing the density of emitters through a reduction of cathode size and spacing. The objective of scaling the substrate size from small (less than $30 \mathrm{~cm}^{2}$ ) to large (greater than $500 \mathrm{~cm}^{2}$ ) areas further compounds manufacturing requirements to a point beyond that easily obtained by modifications to the convention for FEC deposition. A new patented approach to design, assemble, and operate a coating system enables FEC deposition over large areas through process control of source divergence coupled to incremental substrate positioning.
\end{abstract}




\section{INTRODUCTION}

Field emission cathodes (FECs) with characteristics of cold emission, low voltage operation, high current density and microscopic size meet the requirements of an electron source for use in vacuum microelectronics. The host structure for a field emission device usually consists of thin film layers. An insulator is sandwiched between two conducting layers, i.e. electrodes. Holes in the the top conducting layer, i.e. the anode, allow for an etching path into the insulating layer to create a cavity. Exposure to a vapor flux, e.g. via electron beam evaporation or collimated sputtering, induces a progressive closure of the cavity entrance producing a cone-shaped (Spindt) cathode.[1-7] Typically, the cathode base is in direct contact with the surface of the bottom conducting layer. Secondary deposition source(s) positioned along a grazing incidence path to the substrate can be used to accelerate hole closure and/or modify the shape of the cathode. To grow the FECs to a uniform height, roughly equivalent to the thickness of the insulating layer, requires applying a coating of uniform thickness. This procedure in turn requires manipulation of the substrates by planetary fixtures, along with long source-to-substrate distances. For example, greater than a $1 \mathrm{~m}$ working distance is needed for 10 $\mathrm{cm}$ or larger diameter wafers in order to establish a less than $10^{\circ}$ divergence of flux from the evaporation source.

The disproportionate decrease in FEC diameter with respect to height that accompanies a reduction in the desired gate size, from greater than $1 \mu \mathrm{m}$ to less than $0.3 \mu \mathrm{m}$, necessitates a reduction in source divergence in order to prevent premature cavity closure and subcritical cone heights. A conventional deposition approach for "nanocone" FEC formation would require a corresponding increase in source-to-substrate distance. The ensuing, very-tall (greater than $3 \mathrm{~m}$ ) vacuum chambers would only further increase in height with increase in the size of the surface area to be coated. Since equipment costs scale with the amount of surface area to be coated, the large vacuum chamber requirement leads to special-order, high-cost deposition systems. Other limitations of a planetary design include the allowance for vacuum breaks with batch wafer 
processing and overcoming temperature control difficulties that arise due to the substrate transport mechanism. A deposition system is needed to minimize the vacuum chamber dimensions, the source-to-substrate distances and the source divergence while maximizing high quality, sample throughput. A new, alternative approach focuses on the control of the deposition source divergence to uniformly produce FECs not only of nanocone size but over substrates of any size at short source-to-substrate distances.[8]

\section{EXPERIMENTAL DESIGN}

The first consideration is the requirements for tailoring the shape of the cathode with respect to the host substrate structure for low voltage and stable operation. The objective is to obtain an optimal morphology for the shape, height and tip radius of the nanocone. For example, to evaluate if the nanocone performance with a constant aspect ratio of height-to-width (h:w) can be improved through the use of hybrid shapes. Additional consideration to the position of the cathode tip within the gate, i.e. eccentric vs concentric, influences the source-to-substrate geometry as well. In this concept for a deposition system, a rastered coating of pixel segments through a step-and-repeat process minimizes the degree of eccentricity over the substrate surface producing a uniform display. Each pixel across the substrate will have an identical degree of cone eccentricity whereas in a conventional process using planetary substrate fixtures, the eccentricity of each cone positioned within its gate progressively increases towards the edge of the substrate. To determine the effects of deposition geometry on the FEC h:w ratio, consideration is given to the variables of source-to-substrate separation and angular positioning, the divergence of the evaporation source, and the option of depositing a parting layer to facilitate a quick "release" (dissolution) of the cone overcoat layer. Also, flexibility in material selection to enhance stability and minimize voltage degradation may involve a change in the composition 
of the cathode, further modification of the cathode tip composition or ion-irradiation of the cathode surface using an applied substrate bias.

Several system features are needed to provide a new manufactureable process for nanocone cathode deposition. Items to consider include the capability of optimizing cathode shapes at or greater than the throughput of conventional planetary processing. Clearly, it is advantageous to utilize a compact chamber geometry with standard vacuum equipment when establishing the process for cathode deposition over large surfaces areas. In this respect, the new deposition system should allow for a wide range of sample sizes, e.g. substrate edge dimensions of $25 \mathrm{~cm}$ or larger, and the capability to equal or better the typical divergence (e.g. $\left.10^{\circ}\right)$ of conventional, planetary evaporation systems. In the new deposition system, the source-tosubstrate geometry is not governed by the need for high tolerances in coating-thickness uniformity. The goal of nanocone deposition of uniform size and shape over arbitrarily large areas is accomplished through uniform hole closure from the deposition source by the means of uniform source divergence. The divergence of the evaporative flux at the substrate can be controlled through the use of a source-to-substrate aperture. The location of this aperture as well as its size and shape can be modified to yield the desired nanocone size and shape. The aperture will allow for minimization of the source to substrate separation, an increase of deposition rate to more than tens of $\mathrm{nm}(\mathrm{s})$ per second per source which is much greater than achievable with collimated sputtering, and provision that each pixel has the same deposition history prior to hole closure ensuring a uniform eccentricity of tip position. With this approach, cathode deposition using multiple source arrays operating simultaneously leads to further reduction in process time.

The new deposition system is designed using a step-and-repeat process option to utilize control of source divergence for high volume throughput.[8] Control of the source divergence at short source-to-substrate distances requires the substrate surface to be coated in well-defined increments as provided using a 3-axis translation of the substrate (Fig. 1). It is advantageous to coat only the active matrix and not the substrate wafer borders or regions in-between which leads to a natural reduction in process cycle-time, material waste, and down-time cleaning 
requirements. Also, the use of continuously-fed deposition sources allows for reduced service time. Cost benefits of a high throughput process are accessed by continuous wafer shuttling inand-out of vacuum along with substrate handling that allows for temperature controlled heating over short ramping times. Design details and developmental experiments are given in the following section.

\section{RESULTS}

\section{Source Divergence}

The aperture size, shape, and position between the source-and-substrate are varied to affect the FEC morphology. The formulation for determining the full conical angle $(\psi)$ of source divergence is derived from ray tracing the deposition system geometry as follows

$$
\psi=2\left\{\arctan \left[\left(\mathrm{d}_{\mathrm{s}}+\mathrm{d}_{\mathrm{a}}\right)\left(2 \mathrm{~d}_{\mathrm{sa}}\right)^{-1}\right]\right\}
$$

where the source diameter is $d_{s}$, the aperture width is $d_{a}$, and the source-to-aperture separation is $d_{\text {sa }}$. The width of the coated region $\left(d_{c}\right)$ at the substrate then follows as

$$
\mathrm{d}_{\mathrm{c}}=2 \mathrm{~d}_{\mathrm{ss}}\left\{\sin \left[(\psi)(2)^{-1}\right]\right\}-\mathrm{d}_{\mathrm{s}}
$$

where the source-to-substrate separation is $\mathrm{d}_{\mathrm{ss}}$. Formation of the Mo nanocones in cavities of constant size provides an example of aspect ratio, i.e. height (h) to base width (w), variation with source divergence of the full conical angle ( $\psi$ ) (Fig. 2). A simple linear curve fit to the experimental data for $\psi$ (degrees) ranging from $5^{\circ}$ to $30^{\circ}$ yields a correlation coefficient $\mathrm{R}^{2}$ of 0.974 with the following relationship.

$$
(\mathrm{h}: \mathrm{w})=1.670-1.568\left[(\pi \psi)(180)^{-1}\right]
$$

As expected, the nanocone aspect ratio decreases with an increase in source divergence. Other notable conditions during the deposition process leading to the h:w vs $\psi$ plot include a nominal 
substrate temperature of $350 \pm 25^{\circ} \mathrm{C}$, use of a $9.525 \mathrm{~mm}$ diameter rod-fed evaporation source operated at $10 \mathrm{kV}$ and 0.4 amps, apertures that are $9.5-38.1 \mathrm{~mm}$ in width, the positioning of the aperture $6.35 \mathrm{~mm}$ below the substrate, translation speeds of $0.3-8 \mathrm{~mm} \mathrm{sec}^{-1}$, and source-tosubstrate separation of $11.9-20.0 \mathrm{~cm}$. Under these conditions, nanocone deposition is readily achieved over surface areas at rates in excess of $60 \mathrm{~cm}^{2} \mathrm{~min}^{-1}$ per evaporation source. In addition to source divergence, the substrate heating does effect the h:w ratio. Typically, taller cones can be formed with an increase in temperature as the rate of hole closure decreases since the columnar grain size increases.

\section{Temperature Control}

The in-plane motion of the substrate table provides ready access to quick ramping times for temperature-controlled substrate heating. A $5 \mathrm{~cm}$ diameter heater is positioned opposite the evaporation flux, on the reverese-side of the substrate. This provision reduces the requirement of temperature management over the entire substrate to control over just the pixel region being coated which is a significant advantage when large surface areas and substrate sag are factors. As an example, the temperature (T) is measured (Fig. 3) at two points on a Si wafer that are positioned $6 \mathrm{~cm}$ apart and sequentially pass beneath the quartz-lamp heater. The control is the temperature of the heater shroud. At a $1.6 \mathrm{~mm} \mathrm{sec}^{-1}$ translation rate, the substrate rises to the desired temperature $\left(370 \pm 10{ }^{\circ} \mathrm{C}\right)$ coincident with the nanocone deposition. The heater output remains constant and can be controlled to adjust for translation speed and thermal mass.

\section{Nanocone Uniformity and Morphology}

The FECs are deposited in well-defined increments. The process can include the variant of multiple substrate passes to form each nanocone. That is, the FEC does not have to deposited in a single pass of the substrate over the deposition source. The use of multiple substrate passes in combination with different aperture sizes can be used to create hybrid FEC shapes. For instance a very sharp cathode-tip, that is a higher aspect ratio, can be formed on a low aspect 
ratio base to the FEC by changing the source divergence. In the following example, a singlepass formation of each cathode is accomplished with the step-and-repeat process coverage to fill the active matrix at a rate of $25.5 \mathrm{~cm}^{2} \mathrm{~min}^{-1}$ per source. The source-to-substrate separation is $12.7 \mathrm{~cm}$ and the rectangular aperture has a $10 \mathrm{~mm} \times 32 \mathrm{~mm}$ opening. The width of each parallel pass is $11 \mathrm{~mm}$ with an overlap width of $2-3 \mathrm{~mm}$. The $40 \mathrm{~nm}$ thick gate has a diameter of $350 \mathrm{~nm}$ where the dielectric height is $400 \mathrm{~nm}$. The desired aspect ratio of 1.15 is acheived with the $19^{\circ}$ source divergence (referring to Fig. 2) noting that the long axis of the rectangular is used in the direction of the coating pass. The size and shape of the nanocones that result from these deposition conditions are uniform throughout the substrate. For example, scanning electron microscopy images are shown (Fig. 4) as the deposited structure is sampled in cross-section. The nanocone aspect ratio and dimensions are constant at the center of a pass (Fig. 4a) as well as at the edge of a pass (4-5 $\mathrm{mm}$ from the center) where the coated zones overlap across the substrate (Fig. 4b).

The morphology of the nanocone growth is evident in the fracture cross-section seen in Fig. 4c. The columnar microstructure is well defined in this image. Note that the column (grain) size is smaller than the base diameter of the nanocone. This is required in order for the cone shape to form and be controllable through source divergence in a line of sight process. If the column size was competitive with the gate diameter, then a cone shape would not result. Rather, a shape similar to a butte is prevalent as observed for deposits at higher (i.e. greater than $500{ }^{\circ} \mathrm{C}$ ) substrate temperatures. 


\section{SUMMARY}

Fabrication of nanocone FECs is accomplished through control of the deposition source divergence in a step-and-repeat process. The FECs are deposited into the substrate by moving the substrate over the deposition source in well-defined increments. The selection of an aperture size and shape, its relative placement between the source and substrate, and process temperature determine the aspect ratio of the deposited nanocone. The FECs can be fabricated with uniform size and shape over any surface area size. This includes maintaining a uniform eccentricity of FEC tip position within the gate and uniform FEC tip radius. Collimated sputtering or evaporation are suitable as the deposition source(s).[5, 9] The step-and-repeat deposition process provides access to continuous substrate shuttling through the use of vacuum interlocks. The fabrication of FECs at short (in comparison to long) source-to-substrate distances can minimize the size of the deposition chamber, the cost of deposition instrument components, the cost to assemble the deposition system, and the time to process each part.

\section{ACKNOWLEDGMENT}

This work was performed under the auspices of the United States Department of Energy by Lawrence Livermore National Laboratory under contract \#W-7405-Eng-48. 


\section{REFERENCES}

1. C.A. Spindt, “A thin-film field-emission cathode”, J. Appl. Phys. 39 (1968) 3504.

2. C.A. Spindt, I. Brodie, L. Humphrey and E.R. Westerberg, "Physical properties of thin-film field-emission cathodes with molybdenum cones”, J. Appl. Phys. 47 (1976) 5248.

3. C.O. Bozler, C.T. Harris, S. Rabe, D.D. Rathman, M.A. Hollis and H.I. Smith, "Arrays of gated field-emitter cones having $0.32 \mu \mathrm{m}$ tip-to-tip spacing”, J. Vac. Sci. Technol. B 12 (1994) 629.

4. K. Derbyshire, "Beyond AMLCDs: Field Emission displays?”, Sol. Stat. Technol. 37 (11) (1994) 55.

5. G.N.A. van Veen, B. Theunissen, K. van de Heuvel, R. Horne and A.L.J. Burgmans, "Collimated sputter deposition, a novel method for large area deposition of Spindt type field emission tips”, J. Vac. Sci. Technol. B 13 (1995) 478.

6. C.G. Lee, H.Y. Ahn, B.G. Park and J.D. Lee, "New approach to manufacturing field emitter arrays with sub-half-micron gate apertures", J. Vac. Sci. Technol. B 14 (1996) 1966.

7. A.A.G. Driskill-Smith, D.G. Hasko and H. Ahmed, "Fabrication and behavior of nanoscale field emission structures”, J. Vac. Sci. Technol. B 15 (1997) 2773.

8. A.F. Jankowski and J.P. Hayes, "Process System and Method for Fabricating Submicron Field Emission Cathodes”, United States Patent No. 5,746,634, (May 5, 1998).

9. A.F. Jankowski and J.P. Hayes, "Sputter Deposition of Nanocones for Field Emission", in Flat Panel Display Materials III, ed. R. Fulks, G. Parsons, D. Slobodin and T. Yuzuriha, Mat. Res. Soc. Symp. Proc. 471 (1997) 237. 


\section{FIGURE CAPTIONS}

Figure 1. A design schematic of the 3-axis motion, substrate table (top-view above and sideview below) that features (A) stepper-motors, (B) translation drive-shafts, (C) substrate holder, (D) source aperture, and (E) continuously-fed evaporation source.

Figure 2. A decrease in nanocone aspect ratio of height (h) to base (w) follows proportionally to a decrease in the full conical angle $(\psi)$ of source divergence.

Figure 3. Translation of the substrate under a quartz lamp (located opposite the vapor source) provides localized heating for the nanocone deposition. Temperature $\mathrm{T}\left({ }^{\circ} \mathrm{C}\right)$ is monitored at substrate positions (\#1 and \#2) that sequentially pass under the heat lamp that is held at constant temperature.

Figure 4. The nanocones imaged using scanning electron microscopy are shown across two representative, parallel deposition zones. At the (A) center and (B) edge the nanocones have the same aspect ratio and dimensions. (C) A fracture cross-section through a nanocone reveals a columnar growth morphology. 


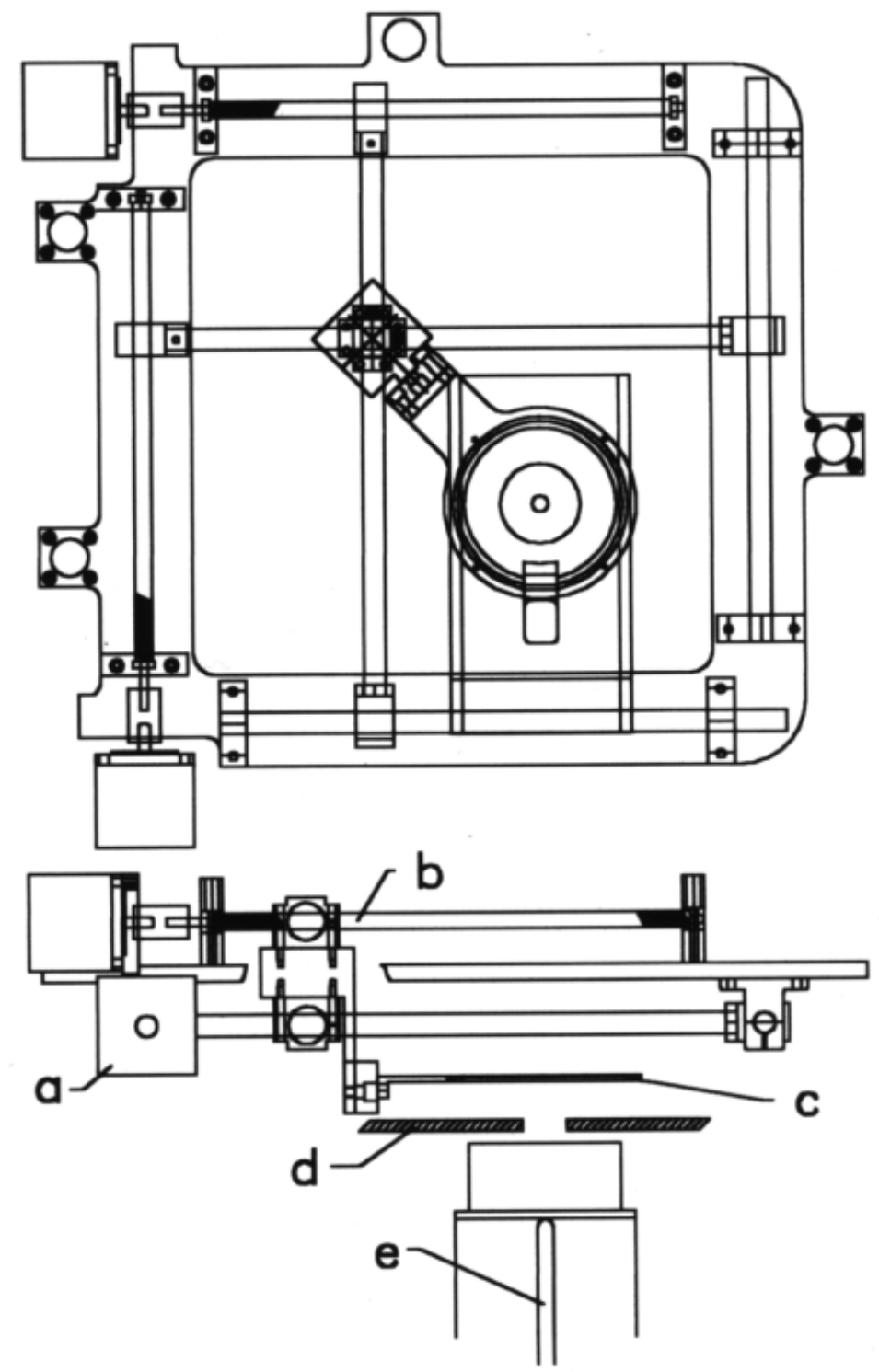

Figure 1. 


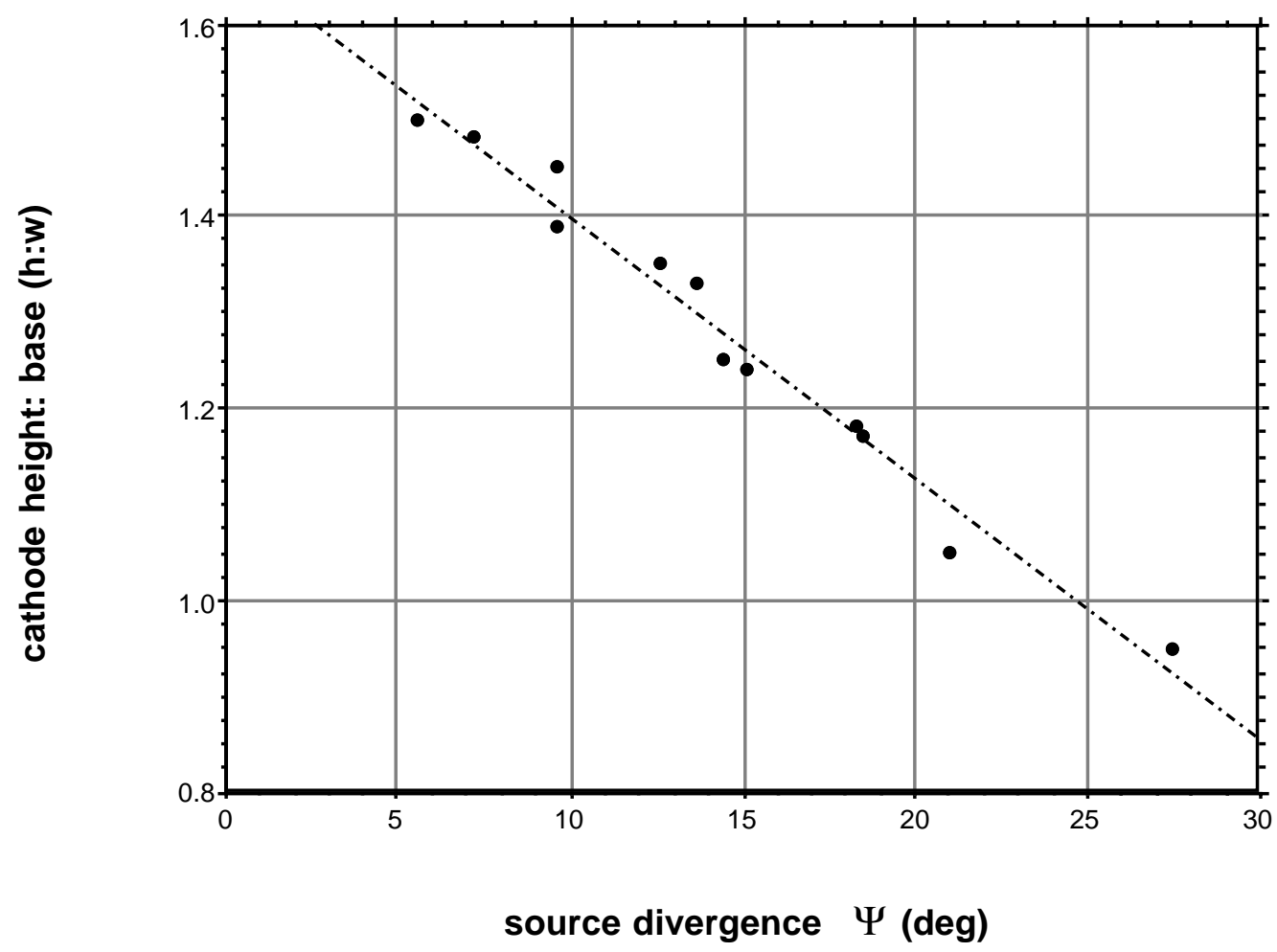

Figure 2. 


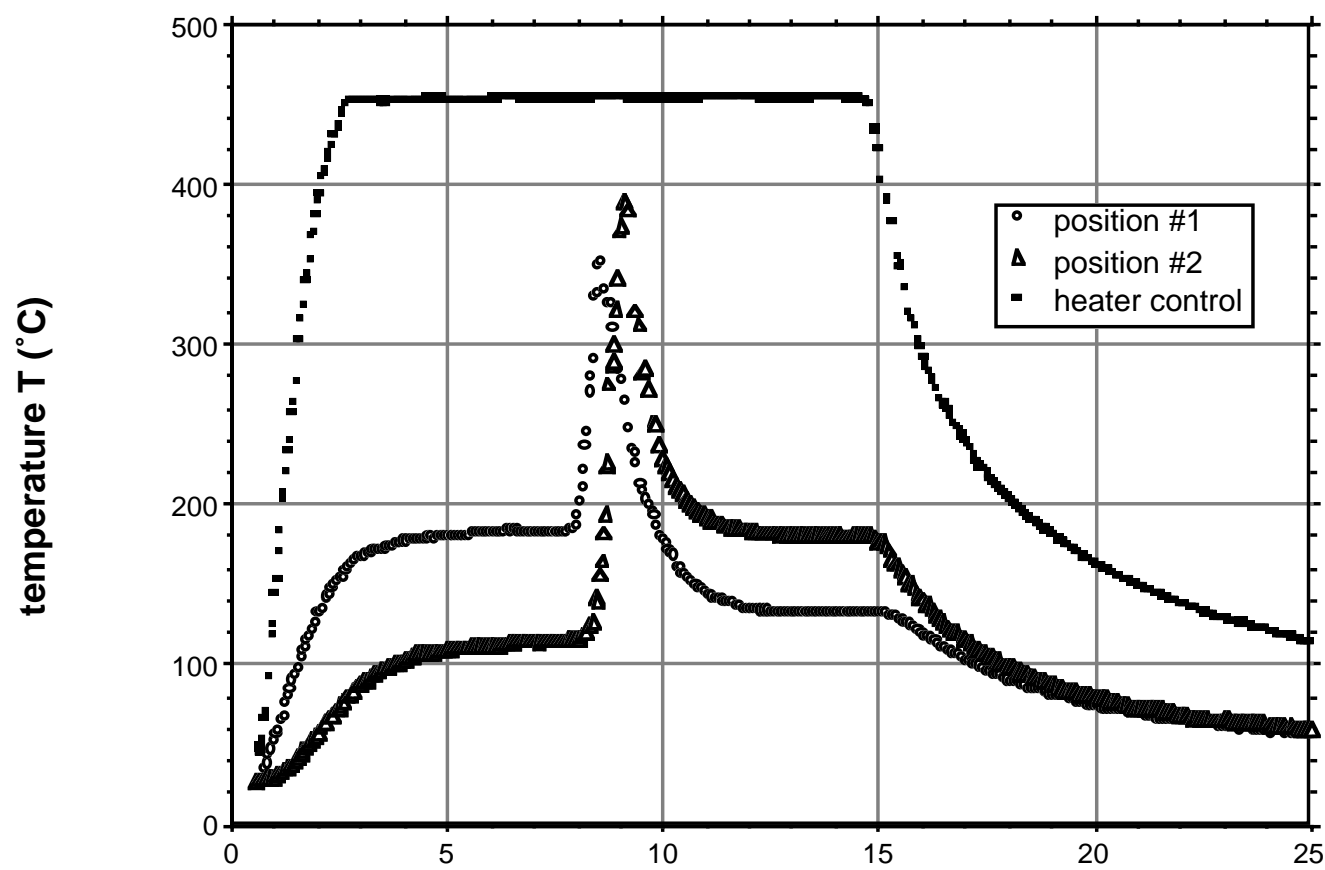

time $\mathbf{t}(\mathrm{min})$

Figure 3. 

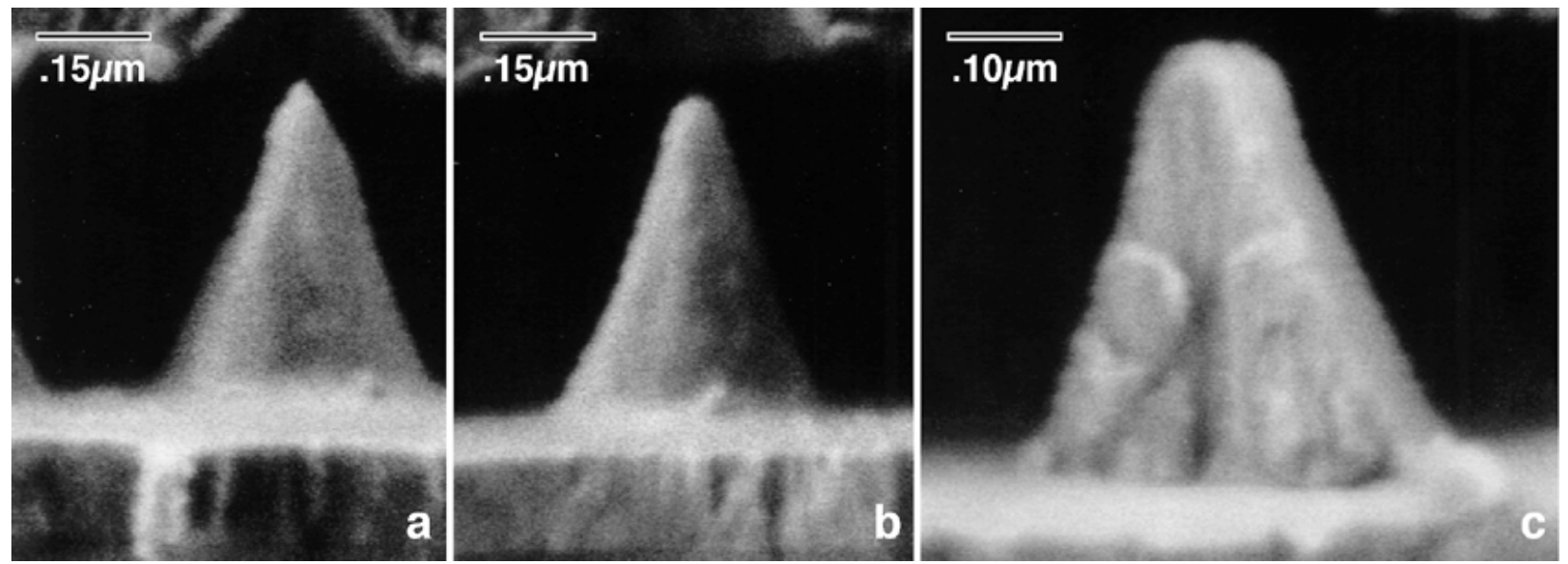

Figure 4. 